\title{
Cirugía proctológica con láser, experiencia inicial en México
}

\author{
Proctological laser surgery, initial experience in Mexico
}

\author{
José Manuel Moreno Berber,* Zaira Eunice Montes Osorio, ${ }^{* *}$ Arturo Mendoza Reyes, ${ }^{* * *}$ \\ María del Rocío Hernández Castro****
}

Palabras clave: Terapia láser, canal anal, fístula anal, cirugía láser, México.

Key words: Laser therapy, anal canal, anal fistula, laser surgery, Mexico.

\author{
Abreviaturas: \\ Láser $\mathrm{CO}_{2}=$ Láser de \\ dióxido de carbono. \\ $\mathrm{Nd}: \mathrm{YAG}=$ Neodymium - \\ doped yttrium aluminium \\ garnet (granate de \\ aluminio e itrio dopado \\ con neodimio). \\ FiLaC = Fistula laser \\ closure (cierre de fístula \\ con láser).
}

* Cirugía de Colon y

Recto. Hospital General

Zona Norte de Puebla,

Secretaría de Salud

del Estado de Puebla.

Hospital Ángeles Puebla.

** Maestría en

Administración de

Servicios y Sistemas

de Salud. Cirujano

General y Laparoscopia

Avanzada. Secretaría

de Salud del Estado

de Puebla. Hospital

Puebla. Hospital Naval

de Alta Especialidad de

Veracruz.

*** Anestesiología.

Hospital Central Militar.

Hospital General

Zona Norte de Puebla,

Secretaría de Salud del

Estado de Puebla.

***** Enfermera

instrumentista. Hospital

Ángeles Puebla.

Recibido: 22/06/2017

Aceptado: 27/10/2017

\section{RESUMEN}

Antecedentes: Las primeras aplicaciones de la energía láser en el tracto gastrointestinal ocurrieron hace apenas una década. Desde entonces, la terapia láser se ha generalizado en el manejo de la enfermedad del colon y recto. En el presente estudio, reportamos la experiencia inicial del uso del láser en el tratamiento de patologías anorrectales en el periodo de enero de 2013 a agosto de 2016 en la ciudad de Puebla, Puebla, México. Material y métodos: Estudio descriptivo, retrospectivo y transversal. Se incluyeron pacientes de los Servicios de Cirugía de Colon y Recto de varios centros hospitalarios de Puebla, Puebla intervenidos con láser en el Hospital General Zona Norte de los servicios de salud del estado de Puebla, Hospital Ángeles Puebla y Hospital Puebla de enero de 2013 a agosto de 2016. Criterios de inclusión: Pacientes con diagnóstico de hemorroides grados II o III, fístula anal, quiste pilonidal no abscedado, condilomatosis perianal y del conducto anal, así como aquellos con fisura anal crónica agudizada. El análisis de las variables fue realizado mediante el programa SPSS versión 22 (IBM). Se expresaron los resultados con estadística descriptiva. Resultados: Se registró una población total de 304 pacientes atendidos con aplicación de láser por padecimiento anorrectal; de ellos, 161 (53\%) fueron varones y 143 (47\%) mujeres. El mayor grupo con patología anorrectal intervenido fue el grupo A, con 187 (61\%) pacientes con enfermedad hemorroidal en quienes se utilizó la técnica HLP; en el caso del grupo D, 14 (5\%) pacientes con diagnóstico de fístulas, empleamos FiLaC, con $14 \%$ de recidiva al tercer mes del postoperatorio. Todos los pacientes se mantuvieron hospitalizados menos de 24 horas por el procedimiento.

\section{ABSTRACT}

Background: The first applications of laser energy in the gastrointestinal tract occurred just a decade ago. Since then, laser therapy has been widespread in the management of colon and rectum disease. In the present study, we report the initial experience of laser use in the treatment of anorectal conditions in the period of January 2013-August 2016 in the city of Puebla, Puebla, Mexico. Material and methods: Descriptive, retrospective and cross-sectional study. We included patients from the Colon and Rectum Surgery Services of several hospitals in Puebla, Puebla, Mexico, with laser treatment at the Hospital General Zona Norte of the health services of the state of Puebla, Hospital Angeles Puebla and Hospital Puebla from January 2013 to August 2016. Inclusion criteria: Patients with diagnosis of grade II or III hemorrhoids, anal fistula, non-abscessed pilonidal cyst, perianal and anal canal condylomatosis, and those with acute chronic anal fissure. The analysis of the variables was by means of the program SPSS version 22 (IBM). Results were expressed using descriptive statistics. Results: We registered a total of 304 patients treated by laser application for anorectal disease, of which 161 (53\%) were men and 143 (47\%) women. The largest group with anorectal involvement was group A, with 187 (61\%) patients with hemorrhoidal disease using the HLP technique; in the case of group D, 14 (5\%) patients with fistula diagnosis, we used FiLaC, with $14 \%$ recurrence at the third postoperative month. All patients were hospitalized less than 24 hours for the surgery.

\section{INTRODUCCIÓN}

$\mathrm{C}$ uando la energía láser interactúa con un tejido, esta es absorbida o dispersada por el mismo dependiendo de varios factores, tales como la conductividad del tejido, la irrigación sanguínea, la duración del pulso láser y la am- plitud de onda de la energía, que determinan la profundidad de penetración hacia el tejido. ${ }^{1}$

Las primeras aplicaciones de la energía láser en el tracto gastrointestinal ocurrieron hace apenas una década. Desde entonces, la terapia láser se ha generalizado en el manejo de la enfermedad del colon y recto como una 
alternativa y nuevo método de tratamiento en trastornos anorrectales. El desarrollo continuo de nuevos sistemas endoscópicos para aumentar la facilidad de acceso al tracto gastrointestinal apunta a aplicaciones cada vez mayores para la terapia con láser en cirugía de colon y recto. ${ }^{2}$ Los tres tipos de láser en uso hoy en día son $\mathrm{CO}_{2}$, argón y Nd:YAG. ${ }^{3} \mathrm{La}$ tecnología láser se ha aplicado ampliamente en el tratamiento de hemorroides, condilomas y neoplasias anorrectales. Sus defensores afirman que produce menos dolor, mejora el proceso de la cicatrización y existe evidencia de una recuperación más rápida en comparación con la cirugía convencional. ${ }^{4,5}$

Se estima que más del $50 \%$ de la población mayor a 50 años de edad ha experimentado problemas de hemorroides. Desafortunadamente, muchas condiciones anorrectales son inapropiadamente atribuidas a hemorroides. Los objetivos de todos los procedimientos para el tratamiento de la enfermedad hemorroidal están basados en tres puntos: 1) disminuir la vascularidad, 2) reducir el tejido redundante y 3) aumentar la fijación hemorroidal a la pared rectal. ${ }^{6}$ La hemorroidectomía con diodo láser cobra importancia para la enfermedad hemorroidal grado II y III, así como también grado IV, pues se ha demostrado que es un procedimiento seguro durante cuya resección no se han presentado complicaciones tempranas ni tardías; esto fue documentado durante un estudio único efectuado en pacientes con enfermedad hemorroidal del Hospital General del Norte de Puebla en México. ${ }^{7}$

La incidencia de fístula perianal reportada en la literatura es de nueve de cada 100,000 habitantes, lo que indica una condición bastante amplia. El tratamiento ideal se basa en tres puntos cardinales: manejo de la infección, cierre del trayecto fistuloso y preservación de la continencia del esfínter. La innovadora técnica FiLaC (por sus siglas en inglés: fistula tract laser closure, cierre de fístula con láser $)^{8,9}$ consiste en el cierre del trayecto fistuloso mediante láser con preservación del esfínter. La intención es proporcionar el cierre al trayecto fistuloso desde su orificio interno hasta el orificio externo; tiene un componente casi indoloro y una reincorporación rápida a la rutina habitual de los pacientes. ${ }^{10,11}$ Giamundo describe una estrategia basada en localizar un setón en el trayecto de la fístula unas semanas antes del tratamiento con láser, lo cual facilita el seguimiento del procedimiento láser y tiene efectos positivos en cuanto a mejorar la cicatrización. ${ }^{12}$ La ausencia de complicaciones, el mínimo dolor postoperatorio y excelentes resultados clínicos han llevado a sugerir una aplicación más amplia de esta técnica. ${ }^{13,14}$

El quiste pilonidal, también conocido como 'absceso pilonidal' o 'sinus pilonidal' es un quiste o absceso cercano a la fisura natal de los glúteos que a menudo contiene pelo y restos de la piel. Las opciones de intervención quirúrgica incluyen incisión con drenaje, electrocirugía y vaporización con láser de dióxido de carbono. A veces es necesario extirpar grandes áreas de la piel, y los defectos pueden ser suturados, cerrados con injertos o manejados como abiertos para la curación por segunda intención. El tratamiento de elección depende de la gravedad, el curso de la enfermedad y la elección del paciente. Se ha probado una combinación de láser de $\mathrm{CO}_{2}$ con láser neodymium-doped yttrium aluminium garnet ( $\mathrm{Nd}$ :YAG) $(1,064 \mathrm{~nm})$ de pulso largo; dicha técnica no necesita anestesia general ni hospitalización. La eliminación de la epidermis y la dermis que contiene los apéndices epidérmicos dañados se hace fácilmente con láser de $\mathrm{CO}_{2}$. Un paciente puede reanudar sus actividades en 4-5 días. ${ }^{15-18}$

Otra patología anorrectal en la que se ha visto la utilidad del uso de láser es el caso de los condilomas. Casi cualquier tipo de láser puede emplearse para resecar lesiones causadas por el virus del papiloma humano; sin embargo, el láser $\mathrm{CO}_{2}$ y el $\mathrm{Nd}$ :YAG son los más comúnmente empleados. El láser emite una longitud de onda en el espectro infrarrojo (10-600 nm). Dado que la absorción total de la energía ocurre en alrededor de $0.1 \mathrm{~mm}$ de la piel, se pueden obtener altos poderes de densidad en pequeños volúmenes de tejido, mientras que un haz enfocado alrededor de $2 \mathrm{~mm}$ de diámetro vaporizará el tejido. Las principales ventajas del láser sobre el bisturí son el perfecto control de la profundidad de la destrucción de la lesión, el riesgo reducido de sangrado y una alta calidad de la cicatrización, dado que la cantidad de necrosis térmica en el tejido sano es mínima. ${ }^{19-21}$ 
En contraparte, la fisura anal, que es una úlcera longitudinal dolorosa en el epitelio escamoso del ano localizada apenas distal a la unión mucocutánea, también puede ocurrir en otras áreas, como la línea media anterior. Las fisuras se pueden clasificar como agudas y crónicas. Una fisura crónica puede ser identificada por la presencia de márgenes indurados, una clara ausencia de tejido de granulación, fibras del esfínter interno visibles en la base de la fisura, un colgajo centinela en el extremo distal de la fisura o un pólipo fibroepitelial en el ápice. Es una patología anorrectal común que afecta a todos los grupos de edad, pero se observa especialmente en adultos jóvenes y sanos, con tasas iguales entre los géneros; generalmente causa dolor en o después de la defecación y durante una a dos horas posteriores a la misma. El tratamiento estándar para la fisura anal es la esfinterotomía interna, que ha demostrado disminuir las presiones internas del esfínter y aumentar el flujo sanguíneo anodermal, pero tiene un riesgo definido de incontinencia fecal permanente. La terapia con láser es una modalidad de tratamiento no invasiva o mínimamente invasiva, segura y eficaz, que puede ayudar a disminuir el dolor, reducir la inflamación y promover la cicatrización de heridas y la reparación de tejidos blandos. El manejo de la fisura anal se basa en aliviar la hipertonicidad anal. Este es un procedimiento simple que puede realizarse con anestesia local en forma ambulatoria, con una mínima morbilidad postoperatoria. ${ }^{22}$

En el presente estudio, reportamos la experiencia inicial que se tiene en México del uso del láser en el tratamiento de patologías anorrectales del año 2013 al 2016 en varios centros hospitalarios de la ciudad de Puebla, México.

\section{MATERIAL Y MÉTODOS}

Estudio descriptivo, retrospectivo y transversal. Todos los pacientes sometidos a procedimientos con láser firmaron consentimiento informado. En el estudio se analizaron un total de 304 pacientes con enfermedades anorrectales, provenientes de tres diferentes hospitales de la ciudad de Puebla, México, en el periodo de enero de 2013 a agosto de 2016, del Servicio de Cirugía de Colon y Recto. Los pacientes fueron intervenidos quirúrgicamente mediante el uso de láser a cargo de un mismo equipo quirúrgico. Se dividieron en cinco grupos de la siguiente manera: grupo $\mathrm{A}$, pacientes con enfermedad hemorroidal sometidos a hemorroidectomía láser; grupo B, pacientes con fisura anal en los que se realizó esfinterotomía lateral interna láser; grupo $\mathrm{C}$, pacientes con condilomatosis perianal y del conducto anal, en quienes efectuamos resección con láser de una o varias lesiones, según fuera el caso; grupo D, pacientes con diagnóstico de fístula anal en los cuales se instauró el cierre con láser del trayecto fistuloso (FiLaC), con colocación de setón ocho semanas previas a la cirugía; y grupo $E$, pacientes con quiste pilonidal, en quienes empleamos la técnica de cierre del sinus pilonidal. Usamos los equipos Ceralas y Leonardo Biolitec ${ }^{\circledR}$ de 980 y 1,470 nanómetros de longitud de onda.

\section{Criterios de inclusión}

- Paciente de la consulta de Cirugía de Colon y Recto.

- Paciente con hemorroides grado II o III.

- Paciente con diagnóstico de fístula anal.

- Paciente con diagnóstico de quiste pilonidal no abscedado.

- Paciente con diagnóstico de condilomatosis perianal y del conducto anal.

- Paciente con diagnóstico de fisura anal crónica agudizada.

- Edad de 15 a 75 años.

Criterios de exclusión

- Paciente con protocolo quirúrgico o expediente incompleto.

- Paciente con disfunción o incompetencia del esfínter.

- Paciente con diagnóstico de cáncer colorrectal.

- Paciente con incontinencia fecal.

Criterios de eliminación

- Paciente con protocolo quirúrgico o expediente incompleto.

- Paciente que no aceptara firmar el consentimiento informado. 
Todos los pacientes fueron evaluados en forma preoperatoria con tacto rectal y proctoscopio; se clasificaron por padecimiento anorrectal presente y de acuerdo con cada grupo del estudio.

\section{Técnica quirúrgica}

Respecto a la técnica operatoria, sin preparación intestinal, los pacientes fueron sometidos a bloqueo regional epidural, colocados en posición de Kraske o navaja Sevillana e intervenidos por el mismo equipo quirúrgico siempre. Para el caso de hemorroidectomías láser, todas fueron realizadas mediante la técnica HLP ${ }^{\circledR}$ (hemorrhoidectomy laser procedure) con fibra de 2.6 metros de longitud; para el tratamiento de las fístulas, se utilizó la técnica FiLaC ${ }^{\circledR}$ (fistula laser closure) en la oclusión láser del trayecto fistuloso con fibra tipo fistula probe de 2.6 metros de longitud (Figuras 1a-1c). La programación fue en modo pulsación entre 10-13 watts con pausas de 0.01 segundos y con una duración de 30 segundos con láser 980 y 1,470 nanómetros. Administramos para la profilaxis preoperatoria ceftriaxona $1 \mathrm{~g}$ y metronidazol $500 \mathrm{mg}$ en forma intravenosa. Para el postoperatorio, no hubo restricción dietética y todos los pacientes se manejaron con ablandador de heces por un periodo de dos semanas, antibioticoterapia oral con metronidazol $500 \mathrm{mg}$ cada ocho horas por cinco días, dieta alta en fibra, baños de asiento y analgésicos en caso necesario. Mediante intervenciones en el expediente clínico y recolección en una base de datos, se obtuvieron las características demográficas de la población en estudio, mientras que los datos de la evaluación clínica se recolectaron durante las consultas de seguimiento.

\section{Seguimiento}

Los pacientes permanecieron hospitalizados durante 24 horas. Fueron evaluados durante la consulta externa después de una semana del tratamiento; luego, a los 14 días, y una cita más al día 30 de su intervención quirúrgica, para finalmente revisarlos al tercer y sexto mes como citas de control. El dolor se evaluó con escala visual análoga del cero al 10 (cero, sin dolor y 10, dolor intenso). También se valoró la presencia de complicaciones para cada uno de los pacientes y de acuerdo al padecimiento anorrectal identificado. Durante la consulta de seguimiento se puntualizó el tiempo en días que tardaron los pacientes en reincorporarse a sus actividades habituales.

\section{Análisis estadístico}

Los datos demográficos se obtuvieron durante el interrogatorio en la primera consulta de valoración preoperatoria y se concentraron en una base de datos que concentraba las variables; se validó su contenido y se les sometió a análisis mediante el programa SPSS versión 22 (IBM Software, Chicago, IL). Se expresaron los resultados mediante estadística descriptiva.
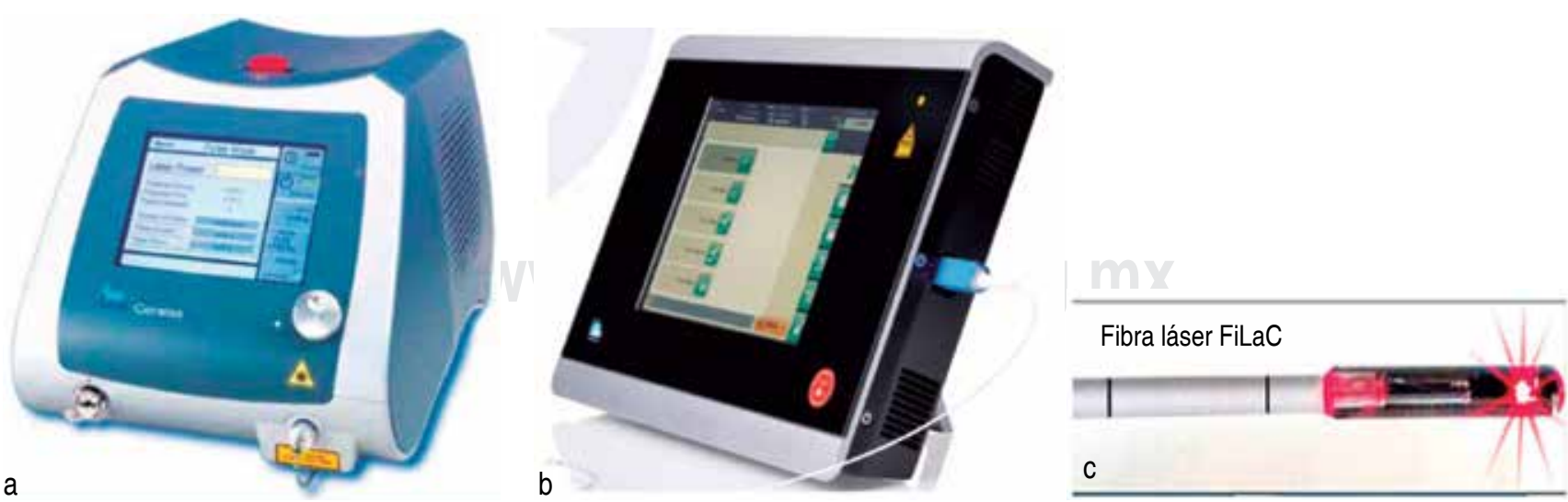

Figura 1. Láser Diodo Ceralas a), y Leonardo Biolitec ${ }^{\circledR}$ b), junto con fibra óptica FiLaC ${ }^{\circledR} \boldsymbol{c}$ ). 


\section{RESULTADOS}

Un total de 304 pacientes fueron sometidos a cirugía láser por padecimientos anorrectales benignos de enero de 2013 a agosto de 2016 por un mismo equipo quirúrgico. Los pacientes que no cumplieron con los criterios de selección no se admitieron en el estudio. El seguimiento abarcó cerca de 12 meses. La media para la edad en el estudio fue de 30.3 años (rango 18-73 años).

De los 304 pacientes estudiados, 161 (53\%) pertenecieron al sexo masculino y 143 (47\%) al sexo femenino (Figura 2).

En cuanto al tipo de diagnóstico: englobados en el grupo A, 187 (61\%) pacientes con enfermedad hemorroidal grado II o III; en el grupo B, 63 (21\%) pacientes con fisura anal; en el grupo $C$, un total de 37 (12\%) presentó un diagnóstico de condilomatosis perianal; en el grupo D, de fístulas anales, 14 (5\%) pacientes, y en el grupo $E$, un total de tres (1\%) pacientes con diagnóstico de quiste pilonidal no abscedado (Figura 3).

Basados en la etiología del padecimiento, se ofreció tratamiento quirúrgico con láser a todos los grupos, como se menciona a continuación: el tratamiento para los pacientes del grupo $A$, con enfermedad hemorroidal, se basó en la técnica HLP, con un tiempo quirúrgico de $22.4 \pm 4.5$ minutos. La intensidad del dolor

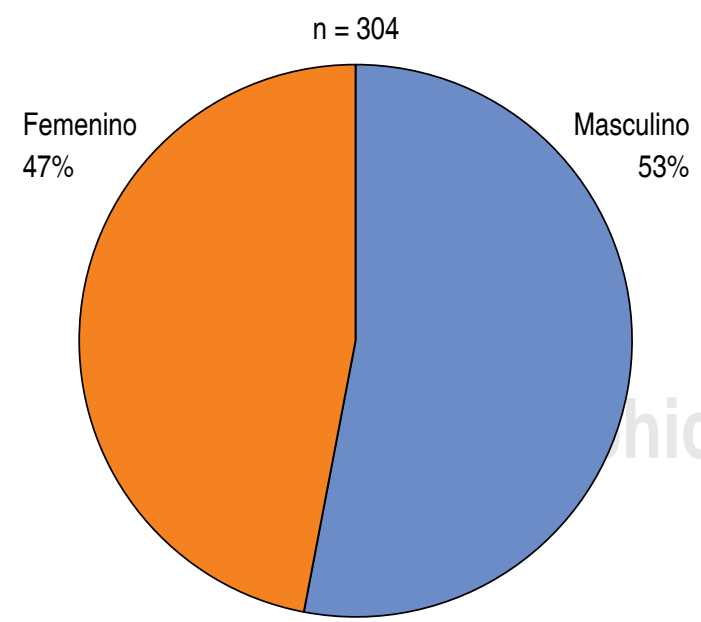

Figura 2. Distribución por sexo de los pacientes del estudio. postoperatorio de acuerdo a la escala visual análoga fue de 3.0 puntos en promedio (1-10) durante la primera semana (Figura 4).

Dentro del grupo B, de fisuras anales, el método quirúrgico efectuado se concentró en la esfinterotomía lateral interna con láser, y en los casos asociados a colgajo centinela (hemorroide centinela), dicho tejido anormal fue resecado también con láser en modo continuo de 10 a 13 watts; todo el procedimiento tomó tan solo $9.22 \pm 1.8$ minutos en promedio.

En el caso del grupo C, la resección de condilomas con láser llevó un tiempo promedio de $11.54 \pm 3.1$ minutos con más de cinco lesiones. Los pacientes no presentaron complicaciones durante el transoperatorio. Todos acudieron a su consulta de seguimiento.

Los pacientes con diagnóstico de fístula anal del grupo $\mathrm{D}$ fueron sometidos a cierre con láser del trayecto fistuloso (FiLaC), contabilizando un tiempo de cirugía promedio de $13.7 \pm 3.3$ minutos por paciente. El total de los 14 pacientes (100\%) de este grupo tuvo un orificio externo y todas las fístulas se clasificaron como transesfintéricas bajas y altas. La longitud promedio del trayecto fistuloso fue de 4.5 centímetros $(2.5-9 \mathrm{~cm})$. No hubo complicaciones en el transoperatorio ni se

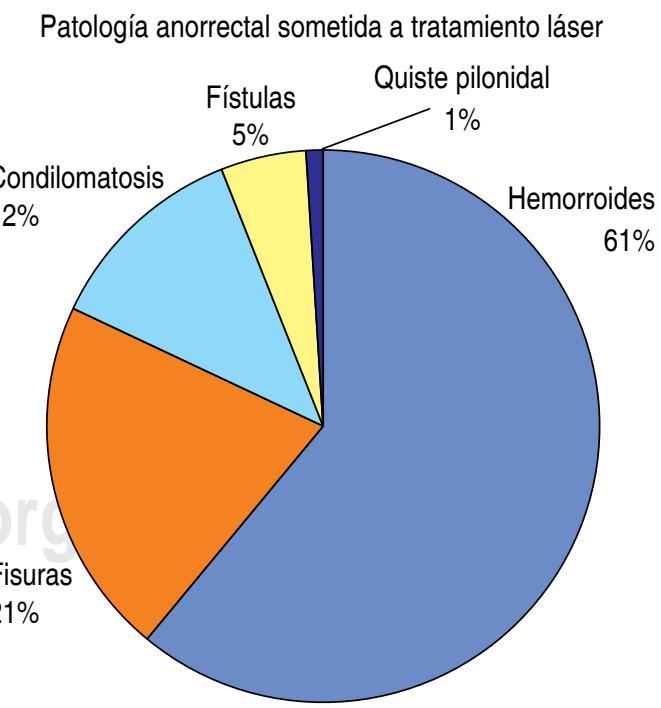

Figura 3. Patología anorrectal encontrada en el estudio. 

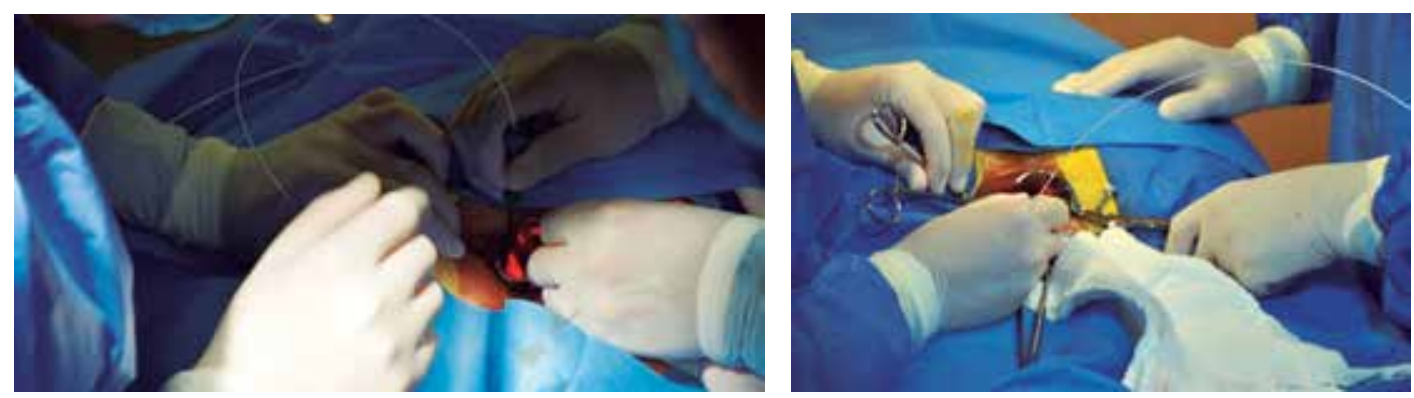

Figura 4. Hemorroidectomía láser (HLP: hemorrhoidectomy laser procedure).
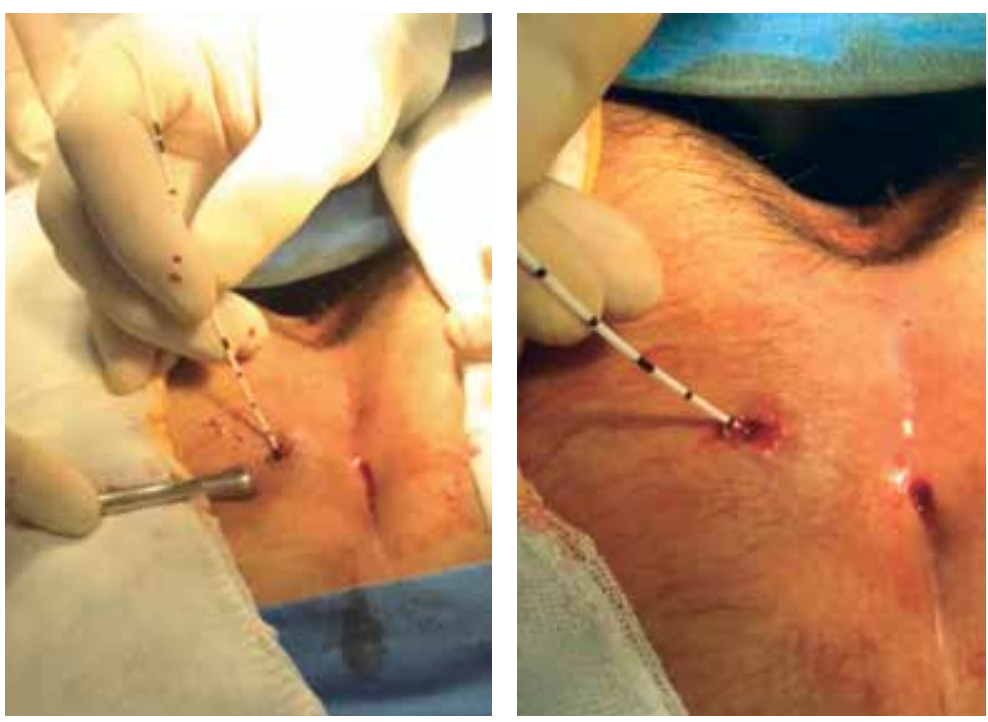

Figura 5. Cierre con láser del sinus pilonidal.

reportaron cambios en la continencia durante el seguimiento postoperatorio.

Para el grupo $E$, de pacientes con quiste pilonidal no abscedado, la duración promedio de la oclusión del sinus pilonidal con láser fue de $15.3 \pm 1.5$ minutos (Figura 5).

Lo referente a la presencia de complicaciones se describe a continuación. En el grupo A, de enfermedad hemorroidal, de un total de 187 pacientes intervenidos con hemorroidectomía láser, en el 2\% (tres pacientes) se identificó clínicamente la presencia de colgajo (hemorroide centinela) y en el $1 \%$ (dos pacientes) sangrado de 20 mililitros que no tuvo mayor afectación hemodinámica en el paciente, no requirió reintervención y cedió a la aplicación de hemostático local.
De los 63 pacientes del grupo B, con fisura anal, el 2\% (un paciente) debutó con absceso postoperatorio, el cual fue manejado con antibioticoterapia a base de metronidazol $500 \mathrm{mg}$ cada ocho horas en forma oral por una semana, con lo que se logró una adecuada evolución, sin recidiva.

En el grupo D, que engloba las fístulas anales de origen criptoglandular, sólo se presentó recidiva en dos (14\%) de los pacientes, que fueron intervenidos con cierre con láser del trayecto fistuloso $\left(\mathrm{FiLaC}^{\circledR}\right)$; la recidiva se identificó a los tres meses del postoperatorio. El uso de setón colocado ocho semanas previas a la fistulotomía favoreció identificar adecuadamente el trayecto de la fístula y logró mejorar el proceso de cicatrización.

En cuanto los grupos $\mathrm{C}$, de condilomatosis, y $E$, de quiste pilonidal, no se presentaron complicaciones durante el tiempo de seguimiento de los pacientes.

Todos los participantes del estudio permanecieron menos de 24 horas hospitalizados. El $100 \%$ de la población analizada acudió a sus citas de control y seguimiento en tiempo y forma. Ninguno de los intervenidos mediante procedimiento láser mostró incontinencia fecal ni urinaria en forma postoperatoria.

\section{DISCUSIÓN}

La técnica $\mathrm{HLP}^{\circledR}$ se encuentra bien establecida ya para el tratamiento de la enfermedad hemorroidal grados I y II, y, en algunos casos, para el grado III, donde el prolapso no es total. Dicha técnica ha sido extendida en forma exitosa en el Reino Unido, Italia, la República Checa, España, Turquía, México y Argentina, entre otros países. 
A pesar de la experiencia descrita en este trabajo, son necesarias nuevas publicaciones con muestras más grandes y un seguimiento más largo, siempre con el objetivo de lograr la excelencia en el tratamiento de estos casos. ${ }^{7,23}$

Giamundo y sus colaboradores, en un estudio comparativo entre la ligadura y la hemorroidectomía láser para las enfermedades hemorroidales de grados II y III mostró para la cirugía con láser menor dolor postoperatorio $(p$ $<0.001$ ), mayor resolución de los síntomas ( $\mathrm{p}$ $<0.001)$ y mejor calidad de vida $(p=0.002)$. Por lo tanto, aunque es una técnica de mayor costo, tiene resultados muy satisfactorios a corto y largo plazo. ${ }^{21}$

Maloku $^{24}$ recalca la preferencia de la hemorroidectomía láser en comparación con la cirugía convencional, ya que el dolor postoperatorio es mucho menor y acorta tiempos quirúrgicos, como lo menciona en su reporte. ${ }^{24}$

Giamundo y su equipo ${ }^{12}$ desarrollaron un estudio retrospectivo utilizando diodo láser con una longitud de onda de 1,470 nm para el tratamiento de fístulas anorrectales mediante la técnica de cierre con láser del trayecto fistuloso $\left(\mathrm{FiLaC}^{\circledR}\right)$; reportaron una de las series más grandes documentadas en la literatura médica, con excelentes resultados, ya que no hubo complicaciones postoperatorias como la incontinencia; también recomendaron la previa colocación de setón para este procedimiento.

La terapia láser es aplicada para estimular la cicatrización y disminuir el dolor; es efectiva para el manejo de las heridas abiertas. Algunos de los efectos conocidos del láser se enfocan en la producción del ATP, el cual actúa como desencadenante en los efectos de bioestimulación, propicia una adecuada microcirculación y acelera el proceso de cicatrización. También se sabe que impacta en el incremento del diámetro de las fibras de colágeno en modelos experimentales en ratas. ${ }^{25}$

Pese a las series que se han efectuado en cuanto al uso de láser en dermatología, cirugía vascular y coloproctología, aún quedan espacios por llenar en las recomendaciones del tipo de láser y la óptima longitud de onda, así como los parámetros del equipo con diodo láser, para obtener resultados que impacten positivamente en la calidad de vida del paciente y en la resolución de su padecimiento.
El manejo quirúrgico de padecimientos anorrectales como la enfermedad hemorroidal, las fisuras anales, los condilomas, las fístulas anales y el quiste pilonidal no abscedado, entre otros, mediante cirugía con láser ha marcado una era totalmente no invasiva, pues son mínimas las complicaciones reportadas, el dolor postoperatorio es menor y hay evidencia de una rápida recuperación de los pacientes en comparación con la cirugía convencional.

\section{CONCLUSIONES}

La aplicación de láser en proctología para la resolución de patologías anorrectales es otra alternativa recomendable entre los métodos existentes. Al considerarse un procedimiento de mínima invasión, confiere seguridad en cuanto a la preservación del anodermo y conservación de la integridad del esfínter anal; técnicamente es menos complejo, ya que su manejo es simple, $y$ brinda altas posibilidades de tratamientos exitosos.

En general, la aplicación de láser en proctología es efectiva y significa una opción confiable para nuestros pacientes mexicanos; es accesible y tiene una mínima morbilidad en el tratamiento de padecimientos anorrectales; ofrece una recuperación más rápida, con menor tiempo de convalecencia.

\section{REFERENCIAS}

1. Buchi KN. Endoscopic gastrointestinal laser therapy. West J Med. 1985; 143: 751-757.

2. Buchi KN. Endoscopic laser surgery in the colon and rectum. Dis Colon Rectum. 1988; 31: 739-745.

3. Walfisch S, Ohana N, Charuzi E. Nd:YAG laser for anorectal surgery: initial experience in Israel. Harefuah. 1994; 126: 1-4, 56.

4. Endres JC, Steinhagen RM. Lasers in anorectal surgery. Surg Clin North Am. 1994; 74: 1415-1432.

5. Skobelkin OK, Brekhov EI, Ul'ianov VI, Tolstykh PI, Kochurkov NV, Sten'ko VG, et al. Experience in the use of laser instruments in surgery of the anorectal region. Khirurgiia (Mosk). 1989; (10): 65-68.

6. Rivadeneira DE, Steele SR, Ternent C, Chalasani S, Buie WD, Rafferty JL, et al. Practice parameters for the management of hemorrhoids (revised 2010). Dis Colon Rectum. 2011; 54: 1059-1064.

7. Montes-Osorio ZE, Moreno-Berber JM. Hemorroidectomía con láser, experiencia del Hospital General Norte de Puebla. Rev Mex Cirug Apar Diges. 2016; 5: 10-15. 
8. Giamundo P, Geraci M, Tibaldi L, Valente M. Closure of fistula-in-ano with laser-FiLaC ${ }^{\mathrm{TM}}$ : an effective novel sphincter-saving procedure for complex disease. Colorectal Dis. 2014; 16: 110-115.

9. Limura E, Giordano P. Modern management of anal fistula. World J Gastroenterol. 2015; 21: 12-20.

10. Quaresimini G, Rossi G. Laser surgery of anal fistulae. Chirurgia. 2016; 29: p. 163-168.

11. Wilhelm A, Fiebig A, Krawczak M. Five years of experience with the FiLaC ${ }^{\mathrm{TM}}$ laser for fistula-in-ano management: long-term follow-up from a single institution. Tech Coloproct. 2017; 21: 269-276.

12. Giamundo P, Esercizio L, Geraci M, Tibaldi L, Valente M. Fistula-tract laser closure $\left(\mathrm{FiLaC}^{\mathrm{TM}}\right)$ : long-term results and new operative strategies. Tech Coloproctol. 2015; 19: 449-453.

13. Bodzin JH. Laser ablation of complex perianal fistulas preserves continence and is a rectum-sparing alternative in Crohn's disease patients. Am Surg. 1998; 64: 627-631; discussion 632.

14. Sica GS, Di Carlo S, Tema G, Montagnese F, Del Vecchio Blanco G, Fiaschetti $V$, et al. Treatment of perianal fistula in Crohn's disease. World J Gastroenterol. 2014; 20: 13205-13210.

15. Jain $V$, Jain $A$. Use of lasers for the management of refractory cases of hidradenitis suppurativa and pilonidal sinus. J Cutan Aesthet Surg. 2012; 5: 190-192.

16. Ghnnam WM, Hafez DM. Laser hair removal as adjunct to surgery for pilonidal sinus: our initial experience. J Cutan Aesthet Surg. 2011; 4: 192-195.

17. Badawy EA, Kanawati MN. Effect of hair removal by $\mathrm{Nd}$ :YAG laser on the recurrence of pilonidal sinus. J Eur Acad Dermatol Venereol. 2009; 23: 883-886.

18. Speck NM, Boechat KP, Santos GM, Ribalta JC. Treatment of Bartholin gland cyst with $\mathrm{CO} 2$ laser. Einstein (Sao Paulo). 2016; 14: 25-29.

19. Madrigal de la Campa MA, Ruiz-Moreno JA, PalaciosOchoa J. Tratamiento de condiloma acuminados gigantes de la vulva combinando láser $\mathrm{CO} 2$ y electrocirugía. Ginecol Obstet Mex. 2000; 68: 27-30.

20. Chen K, Chang BZ, Ju M, Zhang XH, Gu H. Comparative study of photodynamic therapy vs. $\mathrm{CO} 2$ laser vaporization in treatment of condylomata acuminata: a randomized clinical trial. $\mathrm{Br} J$ Dermatol. 2007; 156: 516-520.

21. Chang GJ, Welton ML. Human papillomavirus, condylomata acuminata, and anal neoplasia. Clin Colon Rectal Surg. 2004; 17: 221-230.

22. Esfahani MN, Madani G, Madhkhan S. A novel method of anal fissure laser surgery: a pilot study. Lasers Med Sci. 2015; 30: 1711-1717.

23. Boarini P, Rodriguez-Boarini L, Passo-Candelaria P, Mesquita-Lima E, Rodriguez-Boarini M. LASER hemorrhoidal dearterialization. J. Coloproctol (Rio J). 2017; 37: 38-43.

24. Maloku H, Gashi Z, Lazovic R, Islami H, JunikuShkololli A. Laser hemorrhoidoplasty procedure vs. open surgical hemorrhoidectomy: a trial comparing 2 treatments for hemorrhoids of third and fourth degree. Acta Inform Med. 2014; 22: 365-367.

25. Brightman L, Goldman MP, Taub AF. Sublative rejuvenation: experience with a new fractional radiofrequency system for skin rejuvenation and repair. J Drugs Dermatol. 2009; 8: s9-s13.

Correspondencia:

Dr. José Manuel Moreno Berber

Hospital Ángeles Puebla.

Cirugía de Colon y Recto.

Consultorio 825,

Av. Kepler Núm. 2143,

Reserva Territorial Atlixcáyotl,

72190, Puebla, México.

E-mail: jmanuel.morenobr@gmail.com/

drazairaeunice@gmail.com 